\title{
Molecular characterization and pathogenicity of Erwinia spp. associated with pineapple [Ananas comosus (L.) Merr.] and papaya (Carica papaya L.)
}

\author{
Kogeethavani Ramachandran, Uyub Abdul Manaf², Latiffah Zakaria²*
}

\author{
${ }^{1}$ Rice and Industrial Crops Research Centre, MARDI Seberang Perai, P.O. Box 203, 13200 Kepala Batas, Penang, Malaysia \\ ${ }^{2}$ School of Biological Sciences, Universiti Sains Malaysia, 11800 USM, Penang, Malaysia
}

Received: January 17, 2015

Accepted: October 30, 2015

\begin{abstract}
The Erwinia species are well-known pathogens of economic importance in Malaysia causing serious damage to high-value fruit crops that include pineapple [Ananas comosus (L.) Merr.] and papaya (Carica papaya L.).The 16S rRNA sequence using eubacteria fD1 and rP2 primers, identified two bacteria species; Dickeya zeae from pineapple heart rot, and Erwinia mallotivora from papaya dieback. Phylogenetic analysis based on the neighbor-joining method indicated that all the bacterial isolates clustered in their own taxa and formed monophyletic clades. From the pathogenicity test, all isolates of D. zeae and E. mallotivora showed pathogenic reactions on their respective host plants. Genetic variability of these isolates was assessed using repetitive sequence-based PCR (rep-PCR) fingerprinting. The results indicated interspecies, and intraspecies variation in both species' isolates. There were more polymorphic bands shown by rep-PCR fingerprints than enterobacterial repetitive intergenic consensus (ERIC) and BOX- PCRs, however both species' isolates produced distinguishable banding patterns. Unweighted pair-group method with arithmetic averages (UPGMA) cluster analysis indicated that all Dickeya and Erwinia isolates from the same species were grouped in the same main cluster. Similarity among the isolates ranged from 77 to $99 \%$. Sequencing of $16 \mathrm{~S}$ rRNA using eubacteria fD1 and rP2 primers, and rep-PCR fingerprinting revealed diversity among Dickeya and Erwinia isolates. But this method appears to be reliable for discriminating isolates from pineapple heart rot and papaya dieback.
\end{abstract}

Key words: Erwinia, Dickeya, pineapple, papaya, $16 \mathrm{~S}$ rRNA, rep-PCR

\section{Introduction}

Fruits are an important commodity in Malaysia providing revenue to the local and export markets. However, production has been affected by the serious damage caused by the Erwinia species, particularly bacterial heart rot disease caused by Dickeya zeae previously known as Erwinia chrysanthemi. Bacterial heart rot disease is regarded as one of the most devastating diseases of the pineapple [Ananas comosus (L.) Merr.] crop (Lim 1985; Kaneshiro et al. 2011). Malaysian hybrid pineapple cultivar Josapine has been found to be seriously infected by the disease. Recently, the introduced pineapple cultivar MD2 has also been seriously infected. The symptoms appeared as water soaked lesion arising from the basal, bloated, and darker, infection border on the plant leaves. Another important disease is bacterial dieback attributable to E. mallotivora, causing severe losses to the papaya (Carica papaya L.) crop. The disease symptoms are described as greasy, water-soaked lesions or spots on the fruit and stem (Amin et al. 2011).

Dickeya, a new genus was proposed by Samson (2005) with several new species for E. chrysanthemi. Both Dickeya dadantii and $D$. zeae species were reported as synonyms to E. chrysanthemi strains from biovar 3 and 8, respectively, in pineapple host plant. Strains from pineapple in Malaysia were found closely related to D. zeae from a phylogenetic study on Dickeya spp. using multilocus sequence analysis (Marrero et al. 2013). Due to considerable confusion from the naming and re-naming of the species in the literature, and since D. zeae has often been used as the preferred genus name in the recent literature, $D$. zeae will be used for E. chrysanthemi throughout this manuscript.

The presence and emergence of numerous species of Dickeya and Erwinia has increased the need for understanding the molecular diversity, interspecies and intraspecies relationship of these bacterial isolates for rapid detection and accurate identification as well as for epidemiological studies. Such information is crucial for establishing and implementing practical disease management strategies to reduce or prevent the production and spread of the Dickeya and Erwinia species. Knowledge about the existence of variability in the pathogen population is also important for plant breeding and the resulting improvement programs. 
Very limited studies have been done on the molecular characterization of Dickeya and Erwinia species causing diseases on fruit crops in Malaysia. Therefore, this study was conducted to evaluate genetic diversity among Dickeya and Erwinia isolates obtained from diseased pineapple and papaya in Peninsular Malaysia. This study used the pathogenicity test, $16 \mathrm{~S}$ rRNA sequencing, and rep-PCR fingerprinting.

\section{Materials and Methods}

\section{Isolation of Dickeya and Erwinia isolates}

Bacterial isolates were obtained from plant parts showing symptoms of bacterial heart rot of pineapple and bacterial dieback of papaya. A total of 46 disease samples of pineapple and 38 disease samples of papaya were collected. There were from 3 to 10 diseased samples from each site depending on the level of disease incidence. The sample collection was conducted from June 2010 to June 2011. The samples were collected from several infected farms. The farms are located in northern and southern Peninsular Malaysia in the state of Kedah, Penang and Johor (Table 1). Tissue from the margins of infected sections was incised using a sterile scalpel. This tissue was submerged in a $0.5 \%$ sodium hypochlorite $(\mathrm{NaOCl})$ solution for $3 \mathrm{~min}$ and rinsed with sterile water twice. The dried tissue and $2 \mathrm{ml}$ of sterile water were then ground in a sterile mortar. After $20 \mathrm{~min}$, the diffusion was spread using a sterile spreader on Nutrient Agar (NA) (Himedia, India) or Luria Bertani agar (LB) (Himedia, India) by dilution series (De Boer and Kelman 2001). Well-separated, single colonies were picked after incubation at $25^{\circ} \mathrm{C}$, for 24 to $72 \mathrm{~h}$. The selected isolates were then sub-cultured at least thrice to confirm purity before being stored in 20\% glycerol at $-20^{\circ} \mathrm{C}$, in Nutrient Broth (NB) (Himedia, India). The isolates were tested for their reactions to several important physiological and biochemical characteristics. These characteristics were selected according to the keys of De Boer and Kelman (2001) as preliminary verification on their genus and species.

\section{$16 S$ rRNA gene sequence analysis}

The bacterial isolates were grown on NB overnight while shaking on a shaker at $25^{\circ} \mathrm{C}$ after which the bacterial cells were taken out and measured at $\mathrm{OD}_{600}$ using a spectrophotometer. If the reading showed $0.8-1$, it is an indicator of mid-log phase. The cells were harvested and pelleted by centrifugation for $1 \mathrm{~min}$ at 13,000 rpm. Total genomic DNA of each strain was extracted using a commercially available DNeasy ${ }^{\circledR}$ Plant Mini Kit (Qiagen, United Kingdom) according to manufacturer's instructions, with the exception being that the procedures were started from the DNA lysis step. The extracted DNA was stored at $-20^{\circ} \mathrm{C}$ until it was required.

Using universal primer for eubacteria fD1 and rP2, a PCR amplification of 16S rRNA was conducted (Weisburg et al. 1991). Amplification reactions were prepared in a total volume of $50 \mu \mathrm{l}$ with the following conditions: $10 \mu \mathrm{l}$ 1X PCR buffer, $6 \mu \mathrm{l} 25$ mM MgCl, $1 \mu \mathrm{l} 10$ mM dNTP mix (Promega, USA), $8 \mu$ primer $\mathrm{fD} 1,8 \mu \mathrm{l}$ primer $\mathrm{rP} 2$, $0.25 \mu \mathrm{l} 5$ unit $\cdot \mu \mathrm{l}^{-1} \mathrm{GoTaq}^{\circledR}$ DNA polymerase (Promega, USA), $0.4 \mu \mathrm{l} 10 \mathrm{ng}$ template DNA and $16.35 \mu \mathrm{l}$ nucleasefree water.

Polymerase chain reaction was carried out in DNA Engine $^{\mathrm{TM}}$ Peltier Thermal cycle model PTC-100 according to the PCR programme recommended by Kwon et al. (1997). The polymerase chain reaction product was analysed by loading a mixture of $5 \mu \mathrm{l}$ of the PCR product and $1 \mu \mathrm{l} 6 \mathrm{X}$ loading dye (Fermentas) in $0.8 \%$ agarose gel; electrophoresis was conducted at $70 \mathrm{~V}$ and $400 \mathrm{~mA}$ for 80 min. Purification of the PCR products was done using QIAquick PCR purification kit (Qiagen, United Kingdom) according to the manufacturer's instructions, and was then sent for sequencing to a service provider.

The pairwise alignment of both the forward and reverse 16S rRNA sequences was done using ClustalW. Manual correction and sequence submission for chimera detection followed, using DECIPHER's Find Chimeras web tool (Wright et al. 2012). A consensus sequence was obtained and compared with other sequences in Genbank using Basic Local Alignment Search Tool (BLAST) to determine maximum identity or to determine the closest match of the consensus sequences.

For phylogenetic analysis, multiple sequence alignments were generated and the tree was inferred by the neighbour-joining method in Molecular Evolutionary Genetic Analysis version 6 (MEGA 6) (Tamura et al. 2011). Gaps and missing data were handled by selecting complete deletion in the analysis preferences window for both methods.

In the phylogenetic analysis, E. chrysanthemi from DSM (NR_117737.2), LMG (NR_119368.1), ATCC (NR_118856.1), D. zeae from CFBP (NR_041923.1), D. dadantii from CFBP (NR_041924.1), E. mallotivora strain from Malaysia (HQ456230), DSM (NR_041974.1), LMG (NR_119363.1), ATCC (NR_112557.1), E. papayae from CFBP (NR_042748.1) from the GenBank were included as reference isolates. Pectobacterium caratovorum subsp. caratovorum from ATCC (NR_118855.1), two local isolates of E. carotovorum subsp. carotovorum from jackfruit (Artocarpus heterophyllus, ECC251 and ECC253) were also included to be used as comparison. For an out-group, Ralstonia solaneacearum (NR_074551.1) was included.

\section{Rep-PCR fingerprinting}

The isolates of $P$. carotovorum subsp. carotovorum from jackfruit (ECC251 and ECC253) were also included in repetitive sequence-based PCR (rep-PCR) fingerprinting. Oligonucleotide primers sequences corresponding to repetitive sequence (REP), enterobacterial repetitive intergenic consensus (ERIC) and BOX elements for DNA amplification, were adapted from Versalovic et al. (1994). The polymerase chain reaction mixture in a volume of $25 \mu \mathrm{l}$ was prepared, containing $5 \mu \mathrm{l} 1 \mathrm{X}$ Colorless GoTaq ${ }^{\mathrm{R}}$ Flexi Buffer, $3 \mu \mathrm{l} 25 \mathrm{mM} \mathrm{MgCl}_{2}, 0.5 \mu \mathrm{l} 0.2 \mathrm{mM}$ dNTPs mix (Promega, USA), $0.25 \mu \mathrm{l} 5$ unit $\cdot \mu \mathrm{l}^{-1} \mathrm{GoTaq}^{\circledR}$ DNA polymerase (Promega, USA), $1.25 \mu \mathrm{l} 0.5 \mu \mathrm{M}$ of each primer, and $0.2 \mu$ l genomic DNA. Distilled water was used as negative control. All PCR amplification was performed in 
a thermal cycler (Model PTC 100, M.J. Research Inc., Watertown, MA) and programmed according to PCR cycles recommended by Versalovic et al. (1994).

At least two repetitions of all the PCR reactions were done to determine the reproducibility of the banding patterns. Polymerase chain reaction products were resolved in $1 \%$ agarose gel, electrophoresis at $80 \mathrm{~V}$ and $400 \mathrm{~mA}$, for $120 \mathrm{~min}$. A standard $1 \mathrm{~kb}$-DNA Ladder (Fermentas, Germany) was used as the molecular size marker. The gels were stained with ethidium bromide, viewed and photographed under a UV transilluminator. The banding patterns were recorded using the Quantity One programme software (Bio-RAD) for analysis.

The rep-PCR fingerprints were analysed by the presence and absence of bands of which the bands in each isolate were observed visually and scored as 1 for presence, and 0 for absence regardless of its intensity to generate a binary matrix. Data generated with the three sets of primers were analysed using NTSYS-pc software (Exeter Software, Biostatistics, Inc., NY, USA) version 2.1 (Rohlf 2000). The similarity coefficient was determined using the similarity program for qualitative data (SIMQUAL). Cluster analysis was performed to construct a tree plot using the unweighted pair-group method with arithmetic averages (UPGMA) in the SAHN program of the NTSYS-pc software.

\section{Pathogenicity test on pineapple and papaya}

All Dickeya isolates from pineapple and Erwinia isolates from papaya plants were assessed individually for pathogenicity. The pineapple and papaya test plants were first prepared in a polybag and placed in the plant house of the School of Biological Sciences, Universiti Sains Malaysia, Penang. Bacterial suspension at a concentration of $1 \times 10^{8} \mathrm{CFU}$ per $\mathrm{ml}$ was prepared and inoculated into healthy 4-month-old test plants.

For pineapple, inoculation was performed on the leaf surface of the plant, based on the method described by Kaneshiro et al. (2008). For inoculation, $0.5 \mathrm{ml}$ of bacterial suspension was inoculated into and over slits made in the midsection of the leaf, using a sterile scalpel. A small cotton saturated with inoculum was placed over the slits, held in place with transparent tape and removed after $24 \mathrm{~h}$.

For the papaya plant, a sterilised syringe was used to inject $0.5 \mathrm{ml}$ of bacterial suspension into the apex of the seedling and covered with a small inoculum saturated cotton (Kelman 1953; Amin et al. 2010). The cotton was held in place by parafilm and removed after $24 \mathrm{~h}$.

Each inoculum was repeated for inoculation with 3 replicates. For the negative control, plants were treated with sterile distilled water. Each test plant was covered with a transparent plastic bag to create a moist condition. A moist condition helps to maintain the plant under high humidity (about $80 \%$ ). The bag was removed after 3 days.

The progression of the disease symptoms was observed and recorded from day 3 , for 1 to 3 weeks. The disease severity on pineapple leaves were scored at 7 days after inoculation based on the scale adapted from scale recommended for soft rot disease on the lily plant (Lee et al. 2006). This was due to the similar measuring of the rot- ted segment of the leaves in both plants. Slight modification was made as follows: 0 meant no visible symptoms; $1-0.5-1.0 \mathrm{~cm}$ of the segment (starting from the inoculated position) rotted; $2-1.0-2.5 \mathrm{~cm}$ of the segment rotted; $3-2.5-4.0 \mathrm{~cm}$ of the segment rotted; $4-$ more than $4 \mathrm{~cm}$ or the whole segment rotted. Bacteria were reisolated from the plant part exhibiting disease symptoms following the bacterial isolation procedure described previously.

\section{Results}

\section{Isolation and characterization of Dickeya and Erwinia isolates}

Forty-nine Dickeya and Erwinia isolates comprising 27 isolates from heart rot of pineapple and 22 isolates from dieback of papaya were successfully isolated using NA and LB media. The bacterial isolates were obtained from infected plant parts, such as leaves, stems, and fruits showing disease symptoms. Polymerase chain reaction products produced by all the isolates were $1,500 \mathrm{bp}$ and all the sequences were found not to be a chimera (reference number 0637470608). Based on the closest match of $16 \mathrm{~S}$ rRNA sequences, all 27 isolates from bacterial heart rot of pineapple were identified as D. zeae. The majority of the bacterial isolates (18 isolates) were identified precisely to species level at more than $97.5 \%$ similarity with D. zeae. For dieback of papaya, all 22 isolates were identified as E. mallotivora. However, only six isolates from dieback of papaya were identified precisely to species level at more than $97.5 \%$ similarity with E. mallotivora. The identification and the percentage of similarity as well as GenBank accession numbers of all the isolates are presented in table 1.

From the neighbour-joining tree, Dickeya and Erwinia isolates were placed in their own taxa. The tree can be divided into two main clades, I and II (Fig. 1). The main clade I comprised $D$. zeae isolates which were clustered in sub-clade A. All 27 isolates of D. zeae formed a separate sub-clade (A) indicating that these isolates represent distinct phylogenetic strains of $D$. zeae from heart rot of pineapple. Two isolates of P. carotovorum subsp. carotovorum were grouped in sub-clade B in the main clade I, closely related to the other soft-rotting species of the $D$. zeae group. All the isolates of E. mallotivora which belong to the necrogenic species were grouped together in the main clade II but formed a separate sub-clade (D). Erwinia papayae was grouped in sub-clade $\mathrm{C}$ in the main clade II. The results also indicated that the E. mallotivora from papaya die back in this study, represent distinct phylogenetic strains. The main clade II was placed more distantly from these soft-rotting clades. The sub-clades indicated a high degree of heterogeneity and there were a notable number of isolates from different locations which grouped together among the isolates of both species.

\section{Pathogenicity test}

Symptoms were observed on pineapple plants within $72 \mathrm{~h}$ after inoculation. Different levels of virulence were noted based on disease severity among the isolates. All 27 
Table 1. BLAST results of $16 \mathrm{~S}$ rRNA sequence and similarity percentages of Dickeya and Erwinia strains isolated from pineapple and papaya

\begin{tabular}{|c|c|c|c|c|c|c|c|}
\hline $\begin{array}{l}\text { Bacterial } \\
\text { code }\end{array}$ & Location & Site & Collection year & Host & Bacterial species & $\begin{array}{c}\text { Sequence } \\
\text { similarity [\%] }\end{array}$ & $\begin{array}{c}\text { GenBank deposited } \\
\text { accession No. }\end{array}$ \\
\hline ECH220 & Kedah & 1 & June 2010 & pineapple & Dickeya zeae & 98 & KF057995 \\
\hline ECH222 & Kedah & 1 & June 2010 & pineapple & D. zeae & 98 & KF057996 \\
\hline ECH223 & Kedah & 1 & June 2010 & pineapple & D. zeae & 98 & KF057997 \\
\hline ECH224 & Kedah & 1 & June 2010 & pineapple & D. zeae & 98 & KF057998 \\
\hline ECH273 & Johor & 2 & June 2010 & pineapple & D. zeae & 98 & KF058005 \\
\hline ECH226 & Kedah & 3 & November 2010 & pineapple & D. zeae & 99 & KF057999 \\
\hline ECH227 & Kedah & 3 & November 2010 & pineapple & D. zeae & 99 & KF058000 \\
\hline ECH229 & Kedah & 3 & November 2010 & pineapple & D. zeae & 99 & KF058001 \\
\hline ECH245 & Johor & 4 & January 2011 & pineapple & D. zeae & 98 & KF058033 \\
\hline ECH247 & Johor & 4 & January 2011 & pineapple & D. zeae & 98 & KF058002 \\
\hline ECH248 & Johor & 4 & January 2011 & pineapple & D. zeae & 96 & KF058003 \\
\hline ECH249 & Johor & 4 & January 2011 & pineapple & D. zeae & 99 & KF058004 \\
\hline ECH274 & Johor & 5 & May 2011 & pineapple & D. zeae & 99 & KF058006 \\
\hline ECH275 & Johor & 5 & May 2011 & pineapple & D. zeae & 98 & KF058007 \\
\hline ECH276 & Johor & 5 & May 2011 & pineapple & D. zeae & 98 & KF058034 \\
\hline ECH277 & Johor & 5 & May 2011 & pineapple & D. zeae & 96 & KF058008 \\
\hline ECH278 & Johor & 5 & May 2011 & pineapple & D. zeae & 95 & KF058009 \\
\hline ECH279 & Johor & 5 & May 2011 & pineapple & D. zeae & 99 & KF058032 \\
\hline ECH280 & Johor & 5 & May 2011 & pineapple & D. zeae & 97 & KF058010 \\
\hline ECH281 & Johor & 5 & May 2011 & pineapple & D. zeae & 94 & KF058011 \\
\hline ECH282 & Johor & 5 & May 2011 & pineapple & D. zeae & 98 & KF058012 \\
\hline ECH283 & Johor & 5 & May 2011 & pineapple & D. zeae & 97 & KF058013 \\
\hline ECH284 & Johor & 5 & May 2011 & pineapple & D. zeae & 94 & KF058014 \\
\hline ECH285 & Johor & 5 & May 2011 & pineapple & D. zeae & 97 & KF058015 \\
\hline ECH287 & Johor & 6 & May 2011 & pineapple & D. zeae & 99 & KF058016 \\
\hline ECH288 & Johor & 6 & May 2011 & pineapple & D. zeae & 98 & KF058017 \\
\hline ЕСН308 & Kedah & 7 & June 2011 & pineapple & D. zeae & 97 & KF058035 \\
\hline EM250 & Penang & 8 & January 2011 & papaya & Erwinia mallotivora & 96 & KF058018 \\
\hline EM252 & Penang & 8 & January 2011 & papaya & E. mallotivora & 97 & KF058019 \\
\hline EM254 & Penang & 8 & January 2011 & papaya & E. mallotivora & 97 & KF058020 \\
\hline EM256 & Penang & 9 & January 2011 & papaya & E. mallotivora & 97 & KF058021 \\
\hline EM257 & Penang & 9 & January 2011 & papaya & E. mallotivora & 97 & KF058022 \\
\hline EM271 & Penang & 9 & January 2011 & papaya & E. mallotivora & 98 & KF058043 \\
\hline EM272 & Penang & 9 & January 2011 & papaya & E. mallotivora & 97 & KF058023 \\
\hline EM290 & Johor & 10 & June 2011 & papaya & E. mallotivora & 96 & KF058024 \\
\hline EM291 & Johor & 10 & June 2011 & papaya & E. mallotivora & 94 & KF058025 \\
\hline EM292 & Penang & 11 & June 2011 & papaya & E. mallotivora & 97 & KF058037 \\
\hline EM293 & Penang & 11 & June 2011 & papaya & E. mallotivora & 98 & KF058026 \\
\hline EM294 & Penang & 11 & June 2011 & papaya & E. mallotivora & 97 & KF058038 \\
\hline EM295 & Penang & 11 & June 2011 & papaya & E. mallotivora & 97 & KF058027 \\
\hline EM296 & Penang & 11 & June 2011 & papaya & E. mallotivora & 96 & KF058039 \\
\hline EM297 & Penang & 11 & June 2011 & papaya & E. mallotivora & 97 & KF058040 \\
\hline EM299 & Penang & 11 & June 2011 & papaya & E. mallotivora & 97 & KF058036 \\
\hline EM300 & Penang & 11 & June 2011 & papaya & E. mallotivora & 96 & KF058041 \\
\hline EM301 & Penang & 11 & June 2011 & papaya & E. mallotivora & 97 & KF058042 \\
\hline EM302 & Penang & 11 & June 2011 & papaya & E. mallotivora & 98 & KF058028 \\
\hline EM303 & Penang & 11 & June 2011 & papaya & E. mallotivora & 98 & KF058029 \\
\hline EM304 & Penang & 11 & June 2011 & papaya & E. mallotivora & 99 & KF058030 \\
\hline EM305 & Penang & 11 & June 2011 & papaya & E. mallotivora & 98 & KF058031 \\
\hline
\end{tabular}




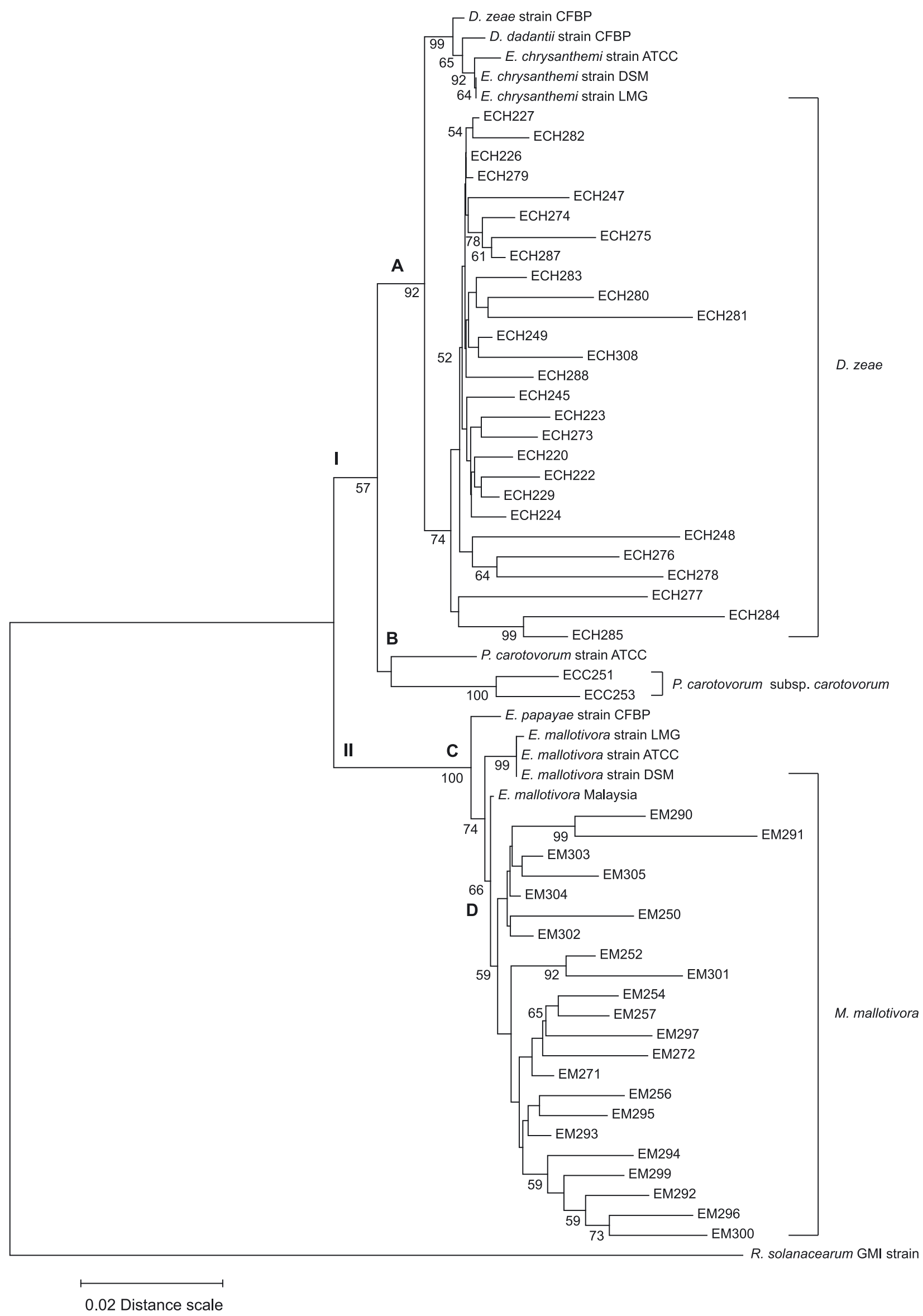

Fig. 1. Neighbour joining tree showing the phylogenetic relationship among pathogenic isolates of Erwinia based on the $16 \mathrm{~S}$ rRNA sequence. Ralstonia solaneacearum (NR_074551.1) is the out-group. I and II - main clade; A, B, C, D - sub-clades 


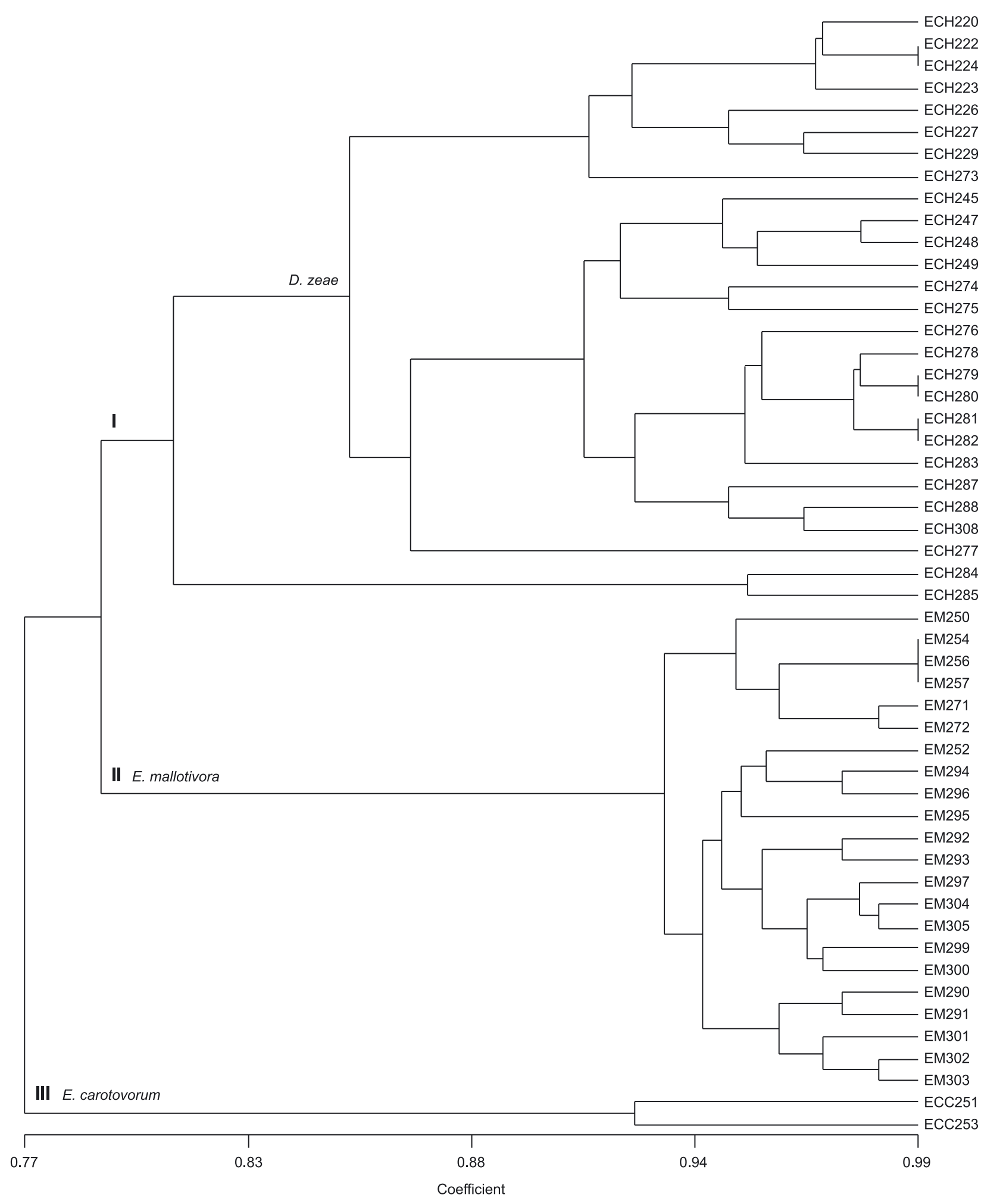

Fig. 2. Dendrogram generated from unweighted pair-group method with arithmetic averages (UPGMA) cluster analysis using simple matching coefficient (SMC) based on combined banding patterns of REP, ERIC and BOX primer sets for 49 Dickeya zeae and Erwinia mallotivora isolates from pineapple heart rot and papaya die back. I, II, III - major clusters

D. zeae isolates produced disease symptoms that initiated as a water-soaked lesion, and bloated and light brown discoloration around the leaf slits, within $72 \mathrm{~h}$ after inoculation. These are indications of a positive reaction for infection as described by Kaneshiro et al. (2008). The water-soaked lesions spread further and may spread to the whole length of the leaf or be arrested around the inoculated region that can be vividly seen as a dark infection border formation. The fully-developed symptoms exhib- ited distinct blisters on the inoculated leaf. Later, the infected area on the leaf started turning light brown to dark brown. At the final stage of the disease, the symptom development was around 21 days after inoculation. At this stage, the whole infected area on the leaf rotted. All the plant leaves artificially inoculated with sterile distilled water as the control, showed no reactions. The extent of disease severity of each of the isolates was measured and scored. The disease score showed 12 isolates at rate 1 , 
seven isolates at rate 2 ; two isolates at rate 3 , and six isolates at 4 , revealing different levels of disease virulence among the isolates. The majority of the isolates showed rate 1 which is a weak pathogenic reaction. Reisolation of $D$. zeae from the leaves showing disease symptoms, confirmed the causal pathogen by physiological and biochemical tests, and Koch's postulate was fulfilled.

Twenty-two isolates of E. mallotivora used in this study showed a positive reaction for infection to papaya plants. Infection appeared as a yellowing of the leaves, dark brown necrosis along the leaf edges followed by a water-soaked and greasy appearance on the bases of the leaf stalks and crown after 4 to 5 days of inoculation. The water-soaked area on the stem eventually spread and later caused rotting of the infected stem, seen as a dark brown to black colouration while leaves slowly wilted and dropped. At the final stage of disease symptom development, the whole stem rot turns black and both leaves and stem collapses, causing dieback or death of the plant after 14 days of inoculation. No disease symptoms were observed on the plant and the leaves that were artificially inoculated with sterile distilled water. All the isolates showed plant death 14 days after inoculation. No disease severity scoring was taken in this study as there was no variation in virulence observed among the isolates. Reisolation of E. mallotivora from the infected plant further confirmed the causal pathogen. Physiological and biochemical tests that completed Koch's postulates were used.

\section{Rep-PCR fingerprinting and cluster analysis}

The amplification of 49 Dickeya and Erwinia isolates with REP, ERIC and BOX primers generated several distinct banding patterns for isolates of D. zeae and E. mallotivora. The major bands obtained for all the isolates varied in size from $350 \mathrm{bp}$ to $4,900 \mathrm{bp}$. The banding patterns with the highest complexity were generated by REP primers with 8 to 15 bands per isolate; ERIC produced 7 to 11 bands while BOX produced 7 to 14 bands. A combined REP, ERIC and BOX-PCRs banding patterns generated a dendrogram that can be divided into three major clusters, I, II, and III, at an $80 \%$ similarity coefficient (Fig. 2). Overall, all isolates were clustered according to their species. Similarity ranged from 77 to $99 \%$. Major cluster I consisted of all isolates of $D$. zeae and the similarity ranged from $81 \%$ to $99 \%$. Major cluster II comprised all isolates of E. mallotivora; the similarity ranged from 93 to $99 \%$. All isolates of P. carotovorum subsp. carotovorum were grouped in major cluster III.

\section{Discussion}

Based on the closest match, D. zeae and E. mallotivora were identified from heart rot of pineapple and die back of papaya, respectively. Phylogenetic analysis using $16 \mathrm{~S}$ rRNA sequences differentiate individual isolates in each species and yielded several clusters within the species exhibiting intraspecies variation. According to Parkinson et al. (2009), isolates or strains that have been present in the environment for a relatively long period might accumulate molecular variation and this may contribute to diversity within a species. This is probably true for E. chrysanthemi. This pathogen has existed in Malaysia for a long time. The pathogen was first reported in 1927 on a pineapple plantation (Johnston 1957; Lim 1985). However, it is a slightly different case for E. mallotivora isolates because the pathogen emergence has been quite recent in Malaysia. The papaya dieback disease was first reported in 2003 by the Department of Agriculture in Johor (southern state) which later spread to many other states of the country. The causal agent was confirmed as E. mallotivora by Amin et al. (2010). Therefore, the diversity observed in E. mallotivora could attribute to an earlier divergence or wider geographical distribution of the pathogen, as described by Avrova et al. (2002)

The soft-rotting D. zeae and P. carotovorum subsp. carotovorum clades were found to be more closely related than the necrogenic species, E. mallotivora clade. This result was similar to the findings reported by Ma et al. (2007), Pitman et al. (2010), and Zhang et al. (2014), indicating an ability to discriminate between the soft-rot and necrogenic species.

The ability to induce disease symptoms on the host plants, together with molecular analysis by $16 \mathrm{~S}$ rRNA sequencing, confirmed the identity of all the isolates as the respective pathogens of pineapple and papaya as described by several investigators (Kaneshiro et al. 2008; Watcharachaiyakup and Kositratana 2009; Amin et al. 2011).

The rep-PCR analysis produced specific fingerprinting profiles that allowed the discrimination of both Dickeya and Erwinia isolates at species level. However, in this study, high polymorphic fingerprinting patterns among isolates within a species were observed in $D$. zeae. The presence of a high numbers of polymorphic patterns for all the primer sets in D. zeae isolates, revealed a high genetic variability among the isolates.

Erwinia mallotivora isolates showed homogenenous fingerprinting patterns, which may reflect the widespread geographic dissemination of these Erwinia isolates (Georghiou et al. 1994). Besides that, the similarly low level of polymorphism observed in several other necrogenic Erwinia groups as E. mallotivora, might also indicate a low incidence of genetic recombinant as reported in these group (Barionovi et al. 2006; Teixeira et al. 2009). Sarkar and Guttman (2004) and Sarkar et al. (2006) likewise suggested it could be due to the narrow host ranges of the species. Thus, it could be that the narrow host range reduced the possibilities for genetic exchange. The opposite was true about the soft rot pathogens Dickeya and Pectobacterium known for having high levels of polymorphism (Gallelli et al. 2009; Nabhan et al. 2012; Ngadze et al. 2012; Terta et al. 2012), and which confirmed the results obtained for D. zeae in this study. Other factors influencing the genetic variability within a pathogen population include movements from different geographic locations or genetic variation due to recombinant or mutation in response to agricultural or environmental constraints (Ochiai et al. 2000).

All the isolates from the same species clustered in the same main clusters, based on the UPGMA cluster analysis using combined rep-PCR analysis of the bands. In the present study, D. zeae isolates showed a high level of intraspecies variation. Soft rot Dickeya and Erwinia are 
known for their wide host range and geographic origins (Smith and Bartz 1990; Seo et al. 2003). So, together with the pathogen, the long existence in this country might have contributed to the isolates' variation. Bacterial dieback caused by E. mallotivora on papaya is a first report and a new disease in Malaysia. Up till now, it is only known to infect papaya plants in Malaysia.

In both trees, Dickeya and Erwinia isolates clustered consistent with their geographical location and time of collection, but no correlations were evident. Similar observations were also reported in soft-rot Erwinia when using amplified fragment length polymorphisms (AFLP) fingerprinting (Avrova et al. 2002). Some of the strains in this study exhibited a close relationship with strains from a different geographical origin within the country. These findings suggest a possible dissemination through contaminated planting material or through irrigation (Teixeira et al. 2009). Isolate ECH284 and ECH 285 were found to be the most divergent group. Isolate ECH277 was the most divergent strain from other $D$. zeae isolates from the same site. Similar results were also obtained for the $16 \mathrm{~S}$ rRNA phylogenetic tree analysis indicating a possible introduction of new strains.

In conclusion, the use eubacteria $\mathrm{fD} 1$ and $\mathrm{rP} 2$ primers for the16S rRNA sequencing and rep-PCR fingerprinting, revealed molecular diversity among Dickeya isolates from pineapple heart rot and Erwinia isolates from papaya dieback in Peninsular Malaysia. Isolates from both species also indicated long-range pathogen dispersal on geographical locations. This information is valuable for early control of disease, planting material quarantine, monitoring pathogen dissemination, and diversity.

\section{Acknowledgements}

This work was supported by the Universiti Sains Malaysia short-term research grant, 304/PBIOLOGI/6311115.

\section{References}

Amin N.M., Bunawan H., Redzuan R.A., Jaganath I.B.S. 2010. Erwinia mallotivora sp., a new pathogen of papaya (Carica papaya) in Peninsular Malaysia. International Journal of Molecular Sciences 12 (1): 39-45.

Avrova A.O., Hyman L.J., Toth R.L., Toth I.K. 2002. Application of amplified fragment length polymorphism fingerprinting for taxonomy and identification of the soft rot bacteria Erwinia carotovora and Erwinia chrysanthemi. Applied and Environmental Microbiology 68 (4): 1499-1508.

Barionovi D., Giorgi S., Stoeger A.R., Ruppitsch W., Scortichini M. 2006. Characterization of Erwinia amylovora strains from different host plants using repetitive-sequences PCR analysis, and restriction fragment length polymorphism and short-sequence DNA repeats of plasmid pEA29. Journal of Applied Microbiology 100 (5): 1084-1094.

De Boer S.H., Kelman A. 2001. Gram-negative bacteria. Erwinia Soft Rot Group. p. 56-72. In: “Laboratory Guide for Identification of Plant Pathogenic Bacteria" (N.W. Schaad, J.B. Jones, W. Chun, eds.). The American Phytopathological Society Press, St. Paul, Minnesota, USA, 398 pp.
Gallelli A., Galli M., De Simone D., Zaccardelli M., Loreti S. 2009. Phenotypic and genetic variability of Pectobacterium carotovorum isolated from artichoke in the Sele Valley. Journal of Plant Pathology 91 (3): 757-761.

Georghiou P.R., Doggett A.M., Kielhofner M.A., Stout J.E., Watson D.A., Lupski J.R., Hamill R.J. 1994. Molecular fingerprinting of Legionella species by repetitive element PCR. Journal of Clinical Microbiology 32 (12): 2989-2994.

Johnston A. 1957. Bacterial heart rots of the pineapple. Malaysian Agricultural Journal 40: 2-8.

Kelman A. 1953. The bacterial wilt caused by Pseudomonas solanacearum. North Carolina Agriculture Experimental Station Technical Bulletin 99: 194.

Kaneshiro W.S., Burger M., Vine B.G., de Silva A.S., Alvarez A.M. 2008. Characterization of Erwinia chrysanthemi from a bacterial heart rot of pineapple outbreak in Hawaii. Plant Disease 92 (10): 1444-1450.

Kwon S.W., Go S.J., Kang H.W., Ryu J.C., Jo J.K. 1997. Phylogenetic analysis of Erwinia species based on 16S rRNA gene sequences. International Journal of Systematic and Evolutionary Microbiology 47: 1061-1067.

Lim W.H. 1985. Diseases and disorders of pineapples in Peninsular Malaysia. MARDI Report 97.

Lee Y.A., Chen K.P., Hsu Y.W. 2006. Characterization of Erwinia chrysanthemi, the soft-rot pathogen of white-flowered calla lily, based on pathogenicity and PCR-RFLP and PFGE analyses. Plant Pathology 55 (4): 530-536.

Ma B., Hibbing M.E., Kim H.S., Reedy R.M., Yedidia I., Breuer J., Charkowski A.O. 2007. Host range and molecular phylogenies of the soft rot enterobacterial genera Pectobacterium and Dickeya. Phytopathology 97: 1150-1163.

Marrero G., Schneider K.L., Jenkins D.M., Alvarez A.M. 2013. Phylogeny and classification of Dickeya based on multilocus sequence analysis. International Journal of Systematic and Evolutionary Microbiology 63 (Pt 9): 3524-3539.

Nabhan S., Wydra K., Linde M., Debener T. 2012. The use of two complementary DNA assays, AFLP and MLSA, for epidemic and phylogenetic studies of pectolytic enterobacterial strains with focus on the heterogeneous species Pectobacterium carotovorum. Plant Pathology 61 (3): 498-508.

Ngadze E., Brady C.L., Coutinho T.A., Van der Waals J.E. 2012. Pectinolytic bacteria associated with potato soft rot and blackleg in South Africa and Zimbabwe. European Journal of Pathology 134 (3): 533-549.

Ochiai H., Horino O., Miyajima K., Kaku H. 2000. Genetic diversity of Xanthomonas oryzae pv. oryzae strains from Sri Lanka. Phytopathology 90: 415-421.

Parkinson N., Stead D., Bew J., Heeney J., Tsror L., Elphinstone J. 2009. Dickeya species relatedness and clade structure determined by comparison of recA sequences. International Journal of Systematic and Evolutionary Microbiology 59 (Pt 10): 2388-2393.

Pitman A.R., Harrow S.A., Visnovsky S.B. 2010. Genetic characterisation of Pectobacterium wasabiae causing soft rot disease of potato in New Zealand. European Journal of Pathology 126 (3): 423-435.

Rohlf F.J. 2000. NTSYSpc: Numerical Taxonomy and Multivariate Analysis System. Version 2.02. Exeter Software, Setauket, New York.

Samson R., Legendre J.B., Christen R., Saux M.F.L., Achouak W., Gardan L. 2005. Transfer of Pectobacterium chrysanthemi 
(Burkholder et al. 1953) Brenner et al. 1973 and Brenneria paradisiaca to the genus Dickeya gen. nov. as Dickeya chrysanthemi comb. nov. and Dickeya paradisiaca comb. nov. and delineation of four novel species, Dickeya dadantii sp. nov., Dickeya dianthicola sp. nov., Dickeya dieffenbachiae sp. nov. and Dickeya zeae sp. nov. International Journal of Systematic and Evolutionary Microbiology 55: 1415-1427.

Sarkar S.F., Guttman D.S. 2004. Evolution of the core genome of Pseudomonas syringae, a highly clonal, endemic plant pathogen. Applied and Environmental Microbiology 70 (4): 1999-2012

Sarkar S.F., Gordon J.S., Martin G.B., Guttman D.S. 2006. Comparative genomics of host-specific virulence in Pseudomonas syringae. Genetics 174 (2): 1041-1056.

Seo S.T., Furuya N., Lim C.K., Takanami Y., Tsuchiya K. 2003. Phenotypic and genetic characterization of Erwinia carotovora from mulberry (Morus spp.). Plant Pathology 52 (2): 140-146.

Smith C., Bartz J.A. 1990. Variation in the pathogenicity and aggressiveness of strains of Erwinia carotovora subsp. carotovora isolated from different hosts. Plant Disease 74 (7): 505-509.

Tamura K., Peterson D., Peterson N., Stecher G., Nei M., Kumar S. 2011. MEGA5: Molecular Evolutionary Genetics Analysis using Maximum Likelihood, Evolutionary Distance, and Maximum Parsimony Methods. Molecular and Biology and Evolution 28 (1): 2731-2739.
Teixeira A.C., Marques A.S., Ferreira M.A. 2009. Low genetic diversity among pathogenic strains of Erwinia psidii from Brazil. Brazilian Journal of Microbiology 40 (3): 678-684.

Terta M., Azelmat S., M'hand R.A., Barakate M., Bouteau F., Ennaji M.M. 2012. Molecular typing of Pectobacterium carotovorum isolated from potato tuber soft rot in Morocco. Annals of Microbiology 62 (4): 1411-1417.

Versalovic J., Schneider M., de Bruijn F.J., Lupski J.R. 1994. Genomic fingerprinting of bacteria using repetitive sequencebased polymerase chain reaction. Methods in Molecular and Cellular Biology 5: 25-40.

Watcharachaiyakup J., Kositratana W. 2009. Metabolic fingerprinting of Erwinia carotovora isolated from jackfruit, chumphada and vegetable. In: The International Society for Southeast Asian Agricultural Sciences Congress 2009, The Emerald Hotel, Bangkok, Thailand, 23-27 February 2009, 213 pp.

Weisburg W.G., Barns S.M., Pelletier D.A., Lane D.J. 1991. 16 S ribosomal DNA amplification for phylogenetic study. Journal of Bacteriology 173 (2): 697-703.

Wright E.S., Yilmaz L.S., Noguera D.R. 2012. DECIPHER, a search-based approach to chimera identification for $16 \mathrm{~S}$ rRNA sequences. Applied and Environmental Microbiology 78 (3): 717-725.

Zhang J., Shen H., Pu X., Lin B., Hu J. 2014. Identification of Dickeya zeae as a causal agent of bacterial soft rot in banana in China. Plant Disease 98 (4): 436-442. 